\title{
Safety of deep slopes excavated during construction of underground structures
}

\author{
P. Procházka ${ }^{1} \&$ V. Doležel ${ }^{2}$ \\ ${ }^{1}$ Czech Technical University in Prague, Czech Republic \\ ${ }^{2}$ Technical University Pardubice, Czech Republic
}

\begin{abstract}
During construction of the Prague subway in the suburbs, a technology of deep slope excavation has been applied. Where excavation is cheaper in comparison to driving is expected, this technology is usable when appropriate conditions are expected on site. From the point of view of the environment, the steeper the slope is, the loss of possible smaller building lots is less. This means that during the construction of the underground structure, the construction of residential buildings and civic amenities can always be in progress in larger areas. Moreover, the excavation of slopes is cheaper the steeper the slope is. The optimization of the slope to be as steep as possible starts with a well known trick given by Prochazka and Koudelka (2001). For given internal parameters an optimal slope can be obtained. On the other hand, if some internal parameter is unknown, it can be a design parameter of the optimization. Similarly, for vertical slopes, the technique of nailed soil can be applied. The optimization in this case is attained from the professional program PLAXIS. This program can be connected with scale modeling on physically equivalent materials. Application to completed construction of one part of the Prague subway will be carried out. The material parameters are given, the process of construction is known, so that a real comparison can be made. The slope of approximately $50 \mathrm{~m}$ height belongs to an exception in the area of applications to the subway construction. Higher slopes are known from tailing dams (deposits of open pit mines), for example, attaining up to $80 \mathrm{~m}$. In the latter case, measurement equipment can be installed in the slope to observe its behavior. This is not the case in our study; the slopes have to be designed in the correct way in order not to fail.
\end{abstract}

Keynotes: deep slopes, damage at interfacial zone, penalty formulation. 


\section{Introduction}

In this paper a problem of minimum stability of deep slopes is solved. In the past, certain approaches have been applied to this problem. One of the most reliable, but simple, is that published in [1]. This procedure enables engineers on site to decide very quickly on how the slope will behave after getting better information on its geological properties. We shortly describe this process in this text and use results from that for comparing them with more precise but much more complicated numerical procedure. The procedure, which we use in this paper, will start with optimization based on the inverse variational principles, [2]. Note that this powerful tool has successfully been applied to concrete problems in [3-5]. In all these cases the optimization can be considered as nonconstrained, only boundary conditions were fulfilled. In our case not only boundary conditions, but also unilateral generalized conditions are used.

The slope is divided into two parts: the stable and the unstable part. The first behaves elastically, as it is well known that the damage behavior is concentrated into a narrow zone in which the slip occurs. The unstable part is simulated by springs; their behavior is also elastic. The springs enable us to come over to penalty-like formulations of the problem.

The generalized Mohr-Coulomb interfacial conditions are taken into account including the tensile stresses on the interface unstable-stable part of the slope to be excluded. The loading is introduced in the classical way: the volume weight is introduced.

The inverse variational principles are naturally connected with finite element method, as shown by Seguchi and Tada [4] and by Tada et al. [5]. But, the FEM is less suitable for the problems involving the optimal shape of boundary because of the division (in any case compulsory using the FEM) of the domain, while the boundary element method seems to be more suitable for such problems. On the other hand, the direct connection with the variational principles is not viewable at first sight. This difficulty will be overcome by the application of Clapeyron's theorem. In the paper, the trick published by Banerjee et al. [6] is developed, concerning the expression of boundary energy in terms of boundary variables.

An example shows a possibility of applications of the theory.

\section{Motivation}

In utilizing the second urban level in urban agglomerations and their neighborhood tendency to realization and optimization of techniques used is observed. The primary effort is seen in the area of maximum decreasing the construction investment and shorting the budget. The tunneling seems to be very expensive in comparison with an open pit excavation and, moreover, the pit should posses its slope as steep as possible (of course, not to avoid the bearing capacity).

Other reasons appear in conjunction with building tunnels for subways. The underground space should involve the second underground traffic level (cars, trains, underpasses for pedestrians) and connecting market rooms, garages, 
stores, and also nontraditional use of underground space for sport reasons. There is a large number of other issues which can be positioned in underground. From this standpoint the underground space is valuable and the entire height from the level of tunnels to the terrain should be exploited. The above said arguments facilitate the usage of open pit technology, if possible to apply.

The pits can profitably be used in the suburb areas where the old buildings are not dense and a construction of new development is expected. This was the case of one part of Prague, for which the study presented in this paper is devoted.

Optimal choice of construction engineering is dependent on many factors, like geological conditions, hydrogeological system, a complex design of the intended structure, traffic communications, utility network, a character of the development in the neighborhood, etc. The main criterion of such a process is the rate of construction and, of course, safety during the construction. In order to fulfill all these criteria pile walls, sheet-pile walls, steel walls, concrete walls, and others are used. The most promising appears to be the slope. In geological conditions where the slopes can be steep, preference is given to this kind of engineering.

This technique is the less used but the most efficient when:

- $\quad$ no estate is present in the site

- the necessary depth is shallow

- $\quad$ the deep level of underground water

- $\quad$ the stability is proved in a reliable way

Very accurate method of verification of stability of slopes is described in this paper. The reliability consists in using two methods with proved eligibility. The first, Apriori Integration, Method, [1], gives the base information on behavior of the slope and the second is very fast numerical method based on finite elements and small movement of slip curve, or surface, from the starting shape according to $[1]$.

\section{Preliminary considerations}

Consider domain $\Omega$ with its boundary $\Gamma$. The internal energy can be expressed as:

$$
\begin{gathered}
(\boldsymbol{\sigma}, \boldsymbol{\varepsilon})=\int_{\Omega} \sigma_{i j} \varepsilon_{i j} \mathrm{~d} \Omega=\frac{1}{2} \int_{\Omega} \sigma_{i j}\left(\frac{\partial u_{i}}{\partial x_{j}}+\frac{\partial u_{j}}{\partial x_{i}}\right) \mathrm{d} \Omega=\frac{1}{2} \int_{\Gamma} \sigma_{i j}\left(u_{i} n_{j}+u_{j} n_{i}\right) \mathrm{d} \Gamma- \\
-\frac{1}{2} \int_{\Omega}\left(\frac{\partial \sigma_{i j}}{\partial x_{j}} u_{i}+\frac{\partial \sigma_{i j}}{\partial x_{i}} u_{j}\right) \mathrm{d} \Omega=\int_{\Gamma} p_{i} u_{i} \mathrm{~d} \Gamma=[\mathbf{p}, \mathbf{u}]
\end{gathered}
$$

where $\boldsymbol{\sigma} \equiv \sigma_{i j}$ is the stress tensor, $\boldsymbol{\varepsilon} \equiv \varepsilon_{i j}$ is the strain tensor, $\mathbf{u} \equiv u_{i}$ is the displacement vector, $\mathbf{p} \equiv p_{i}$ is the traction vector defined on the boundary, $\mathbf{n} \equiv n_{i}$ is the outward unit normal to the boundary $\Gamma$. Symbols (.,.) and [.,.] are respectively internal and external energies. The equation (1) verifies assertion 
that external and internal energies are equal. If necessity of identification of domain $V$ appears, we write $(., .)_{V}$ instead of $(.,$.$) and if the boundary is identified$ by $S$ then $[., .]_{S}$ is written rather then $[.,$.$] .$

The classical principle of minimum potential energy requires that

$$
\frac{1}{2} a(\mathbf{u}, \mathbf{u})-(\overline{\mathbf{b}}, \mathbf{u})-[\overline{\mathbf{p}}, \mathbf{u}] \rightarrow \text { minimum }
$$

where $a(\mathbf{u}, \mathbf{u})=(\boldsymbol{\sigma}(\mathbf{u}), \boldsymbol{\varepsilon}(\mathbf{u}))$ and $\mathbf{b}$ is the volume weight vector. The principle of minimum complementary energy is written as

$$
\frac{1}{2}(\boldsymbol{\sigma}, \boldsymbol{\varepsilon}(\boldsymbol{\sigma}))-[\mathbf{p}, \overline{\mathbf{u}}] \rightarrow \text { minimum }
$$

where barred quantities are given, linear Hooke's law

$$
\sigma_{i j}=L_{i j k l} \varepsilon_{k l} \quad \text { or } \quad \varepsilon_{i j}=C_{i j k l} \sigma_{k l}, \quad \mathbf{L}=\mathbf{C}^{-1}
$$

is fulfilled, statical boundary conditions and kinematical equations are obeyed. In the classical version the principle of minimum potential energy depends only on the choice of the displacement field and prescribed shape of the domain and boundary and given tractions, the principle of minimum complementary energy on the same shape and on prescribed boundary displacements. The idea of the inverse variational principle consists in the fact that also the shape of the domain can change. Using this assumption, one should discover what happens, if such an assumption is accepted.

Splitting the boundary $\Gamma$ into two disjoint parts: $\Gamma_{u}$ where the displacements are prescribed and $\Gamma_{p}$ with prescribed tractions, and using (1), the functionals in (2) and (3) to be minimized turn to the form:

$$
\begin{gathered}
\frac{1}{2}[\mathbf{p}, \mathbf{u}]_{\Gamma_{u}}+\frac{1}{2}[\mathbf{p}, \mathbf{u}]_{\Gamma_{p}}-(\mathbf{b}, \mathbf{u})-[\overline{\mathbf{p}}, \mathbf{u}]_{\Gamma_{p}} \rightarrow \text { minimum } \\
\frac{1}{2}[\mathbf{p}, \mathbf{u}]_{\Gamma_{u}}+\frac{1}{2}[\mathbf{p}, \mathbf{u}]_{\Gamma_{p}}-[\mathbf{p}, \overline{\mathbf{u}}]_{\Gamma_{u}} \rightarrow \text { minimum }
\end{gathered}
$$

After easy algebra the last requirements lead us to

$$
\begin{gathered}
\frac{1}{2}[\mathbf{p}, \mathbf{u}]_{\Gamma_{u}}+\frac{1}{2}[\mathbf{p}-\overline{\mathbf{p}}, \mathbf{u}]_{\Gamma_{p}}-(\mathbf{b}, \mathbf{u})-\frac{1}{2}[\overline{\mathbf{p}}, \mathbf{u}]_{\Gamma_{p}} \rightarrow \text { minimum } \\
\frac{1}{2}[\mathbf{p}, \mathbf{u}]_{\Gamma_{p}}+\frac{1}{2}[\mathbf{p}, \mathbf{u}-\overline{\mathbf{u}}]_{\Gamma_{u}}-\frac{1}{2}[\mathbf{p}, \overline{\mathbf{u}}]_{\Gamma_{u}} \rightarrow \text { minimum }
\end{gathered}
$$


As the second terms in the latter equations disappear $\left(\mathbf{u}=\overline{\mathbf{u}}\right.$ on $\Gamma_{\mathrm{u}}$ and $\mathbf{p}=\overline{\mathbf{p}}$ on $\Gamma_{p}$ ), we eventually get

$$
\begin{gathered}
\frac{1}{2}[\mathbf{p}, \mathbf{u}]_{\Gamma_{u}}-(\mathbf{b}, \mathbf{u})-\frac{1}{2}[\overline{\mathbf{p}}, \mathbf{u}]_{\Gamma_{p}} \rightarrow \text { minimum } \\
-\frac{1}{2}[\mathbf{p}, \overline{\mathbf{u}}]_{\Gamma_{u}}+\frac{1}{2}[\mathbf{p}, \mathbf{u}]_{\Gamma_{p}} \rightarrow \text { minimum }
\end{gathered}
$$

The first condition in (5) and (6) is fulfilled for each admissible $\mathbf{u}$ and the second for all admissible $\mathbf{p}$.

Since $\mathbf{b}$ is given function of position, from the primary principle $\left(6_{1}\right)$ it follows that for fixed $\Gamma_{p}$ the minimum strain energy function is attained along the boundary $\Gamma_{u}$ for which the minimum of the functional is attained. Similarly, from the second (complementary) principle $\left(6_{2}\right)$ we get minimum complimentary energy along such $\Gamma_{p}$ for which the functional reaches its minimum.

\section{Formulation of the problem}

In the above explanation we did not mention one important fact. The solution of the optimization problem has to be fulfilled under additional condition, otherwise no solution exists. In terms of the inverse variational principles constant volume of the optimized domain is assumed.

The problem can then be formulated as: Let the displacement field $\mathbf{u}$ be a solution of a partial differential equation (or, alternatively, $\mathbf{u}$ is a solution of a variational principle) in the domain $\Omega$. Let $E(\mathbf{u}, \Omega)$ be a real function of $\mathbf{u}$ and $\Omega$. The problem of searching for optimal shape consists of finding such a domain from a class $O$ of admissible functions, which minimizes $E$. Note that in the problem of slope stability $O$ is the class of domains with constant measure (in 2D area, volume in 3D). It may symbolically be defined as

$$
\min \{E(\mathbf{u}, \Omega) ; A(\mathbf{u}, \Omega)=0\}
$$

where $A$ is an operator which for each $\Omega \subset O$ uniquely determines the displacement field $\mathbf{u}$.

One of reasonable and practical forms of requirements of designers is an assumption of minimum complementary energy of a structure subject to the volume weight. Such a problem may be formulated in terms of inverse variational principles. In order to ensure the correctness of this formulation, additional constraint conditions have to be applied. As above said, we assume the constant volume of the moving part of the slope under consideration; this is one of the main features of the inverse variational principles.

In other problems other constraints may be applied. Even the $C$ defining the measure of the domain may be made free (no longer constant). Then more 
complicated formulation is expected, involving $C$ as an additional variable in the principle. A typical application of that kind is to the slope stability problems.

The inverse variational principle based on the principle of minimum potential energy constrained by a constant volume of the moving part of the slope can be written in the following form, see (2):

$$
\Pi(\mathbf{u}, \Omega)=\frac{1}{2} a(\mathbf{u}, \mathbf{u})-(\overline{\mathbf{b}}, \mathbf{u})-[\overline{\mathbf{p}}, \mathbf{u}]+\lambda\left(\int_{\Omega} \mathrm{d} \Omega-C\right) \rightarrow \text { minimum }
$$

where $\lambda$ is Lagrange multiplier.

Let us introduce finite elements $\Omega_{i}$ and approximations of the displacements on $\Omega_{i}$ as

$$
u_{i}(\mathbf{x})=\Phi_{i j}(\mathbf{x}) U_{j}, \quad \mathbf{x} \in \Omega_{i}, \quad i=1, \ldots, n, j=1, \ldots, m
$$

where $U_{j}$ denote degrees of freedom of the problem. In the standard way, substituting (9) to (8), one gets

$$
\Pi(\mathbf{u}, \Omega)=\frac{1}{2} K_{i j} U_{i} U_{j}-B_{i} U_{i}-P_{i} U_{i}+\lambda\left(\int_{\Omega} \mathrm{d} \Omega-C\right)
$$

Our aim will now be to formulate the area of the domain by means of its corresponding boundary. This can be done in many ways. For example, suppose the polygonal shape of the structure under study, approximating the moving part of the slope, where the edges of the polygon are created by boundary elements. One can choose some fixed point $P$ (pole) and connect it with each vertex of this polygonal boundary. In this way we obtain $N$ triangles $T_{k}, k=1, \ldots, N$, where $N$ is the number of vertices. Since $\int_{\Omega} \mathrm{d} \Omega=$ meas $\Omega$, i.e. measure of the domain, this measure may then be written as

$$
\operatorname{meas} \Omega=\sum_{k=1}^{N} \operatorname{meas}\left(T_{k}\right)
$$

where meas $\left(T_{k}\right)$ stands for the algebraic measure of $T_{k}, k=1, \ldots, N$. The situation is illustrated in Fig. 1. Determination of the area of any triangle is very simple using vertex coordinates. The triangles their edges are denoted in Fig. 1 as positive are added (with the sign plus) and the triangles with edges denoted as negative are subtracted (sign minus) in the sum (11). The situation is clear from Fig. 1, where a typical area is calculated. 


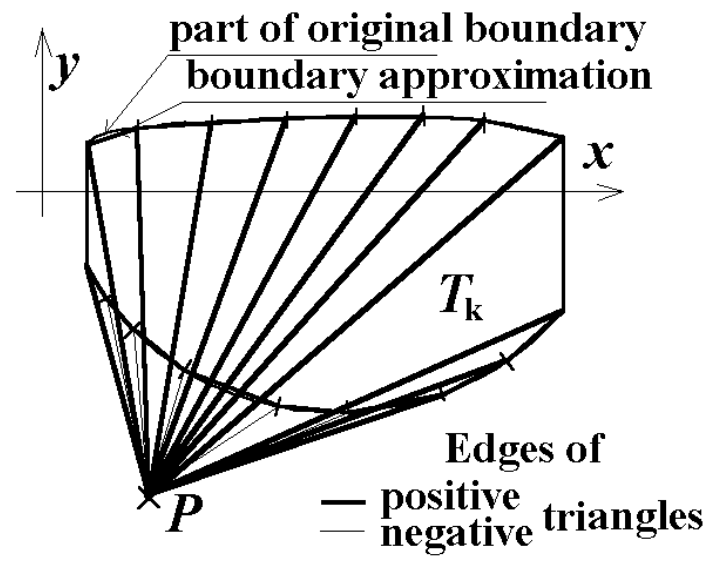

Figure 1: Calculation of the area of a domain.

Now define the shape variables $s_{k}, k=1, \ldots, M$. Taking account of the above arguments, the discretized inverse variational principle is formulated in the following manner:

$$
\Pi\left(\mathbf{u}, T_{k}\right)=\frac{1}{2} K_{i j}(\mathbf{s}) U_{i} U_{j}-B_{i} U_{i}-P_{i} U_{i}+\lambda\left(\sum_{k=1}^{N} \operatorname{meas}\left(T_{k}\right)-C\right) \rightarrow \text { minimum }
$$

where $\mathbf{s}=\left\{s_{1}, s_{2}, \ldots, s_{M}\right\}$ is the vector of shape variables.

\section{Euler's equations}

The stationary requirement leads us to differentiation of $\Pi$ by $U_{k}$ and to the well known expression for finite elements:

$$
\mathbf{K} \mathbf{U}=\mathbf{F} \text {, }
$$

where $\mathbf{K}$ is the stiffness matrix of the system, symmetric and banded. The vector $\mathbf{U}$ involves values of approximations of displacements at degrees of freedom; $\mathbf{F}$ is the vector of influences of external forces, i.e. of volume weight, in our case.

Since $\mathbf{K}$ is dependent of the shape parameters $\mathbf{s}$, we also can differentiate by $s, l=1, \ldots, M$, to get the extreme of the potential energy constrained by constant measure of the admissible domain:

$$
\frac{1}{2} \frac{\partial K_{i k}}{\partial s_{l}} U_{i} U_{k}+\lambda \sum_{k=1}^{N} \frac{\partial \operatorname{meas}\left(T_{k}\right)}{\partial s_{l}}=0, \quad l=1, \ldots, M
$$

or 


$$
E_{l}+\lambda=0, \quad l=1, \ldots, M
$$

where

$$
E_{l}=\frac{\frac{1}{2} \frac{\partial K_{i k}}{\partial s_{l}} U_{i} U_{k}}{\sum_{k=1}^{N} \frac{\partial \operatorname{meas}\left(T_{k}\right)}{\partial s_{l}}}
$$

Equation (15) requires $E_{l}$ to hold its value for any $l$, which is equal to the same constant $-\lambda$. In other words the optimal shape of the trial body will be reached when the strain energy density will be the same at any point on the "moving" boundary of the slope under trial. For this reason it is assumed that the body of the structure should increase its area (in 3D its volume) at the nodal point $l$ of the boundary, $E_{l}$ in which is larger than the true value of $-\lambda$, whilst it should decrease when $E_{l}$ is smaller than the right $-\lambda$. As, probably, we will not know the real value of $-\lambda$ apriori, we estimate it from the average of the current values at the nodal points.

Since $E_{l}, l=1, \ldots, \mathrm{M}$, show large differences in their values, the logarithmic scale were proposed by Seguchi and Tada in [4]. The computational procedure follows this idea.

The variation by $\lambda$ completes the system of Euler's equations:

$$
\sum_{k=1}^{N} \operatorname{meas}\left(T_{k}\right)=C
$$

\section{Application to a real problem}

In order to show the results of the algorithms, one concrete example is considered. Relatively high values of the geomechanical parameters are taken from a real site conditions: $E=400 \mathrm{MPa}, v=0.27$, the cohesion is $10 \mathrm{kPa}$ and thangent of the angle of internal friction is 0.33 . The slope of $45^{\circ}$ was assessed. The starting area of $2 \mathrm{D}$ problem was considered $180 \mathrm{~m}^{2}$. According to [1], the slip curve should meet the toe of the slope. The distribution of contact displacements for bonded slip curve and movement on the contact after introducing Mohr-Coulomb conditions are depicted in Fig. 2. The magnitudes of displacements are much larger in normal direction at the bottom of the slip curve and are suppressed in the case of slip admission.

The optimal slip curve was attained for the area $221 \mathrm{~m}^{2}$ of the domain $\Omega$. The original share $\left(180 \mathrm{~m}^{2}\right)$ and the final are compared in Fig. 3. It is obvious that the difference of shapes of both slopes is very small. This is an impact of very good estimation of the starting shape due to [1]. 
The safety margin $s$ can be defined in the following way: Denote the part of the contact boundary where slip occurs (shear damage) as $\Gamma_{\text {slip }}$ then

$$
s=1-\frac{\operatorname{meas} \Gamma_{\text {slip }}}{\operatorname{meas} \Gamma_{\mathrm{C}}}
$$

where $\Gamma_{\mathrm{C}}$ is the entire boundary and meas is the length. If there is no shear damage on the contact boundary, the safety margin is one and if slip part attains zero, the safety margin is also zero. Note that in our case the safety margin is one and it is 0.8 for the slope of $59^{\circ}$, which was also considered as the optimal.

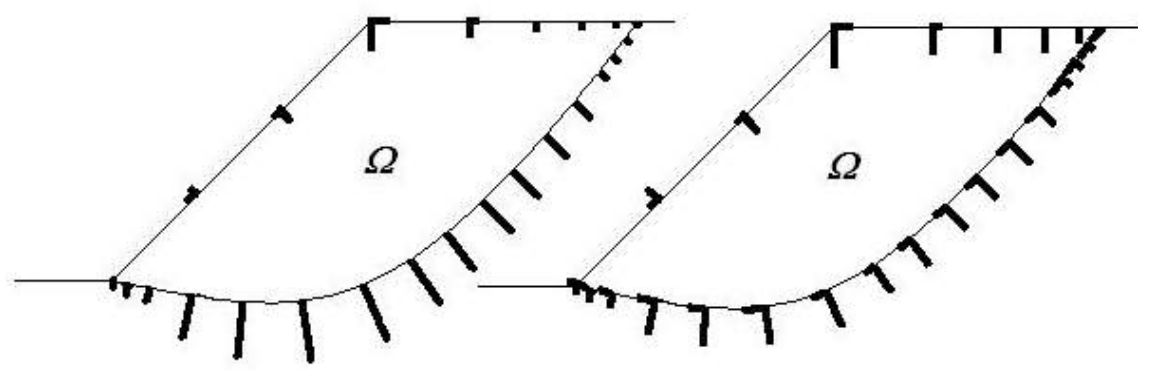

Figure 2: Distribution of displacements on the contact in initial state for bonded and slipped case.

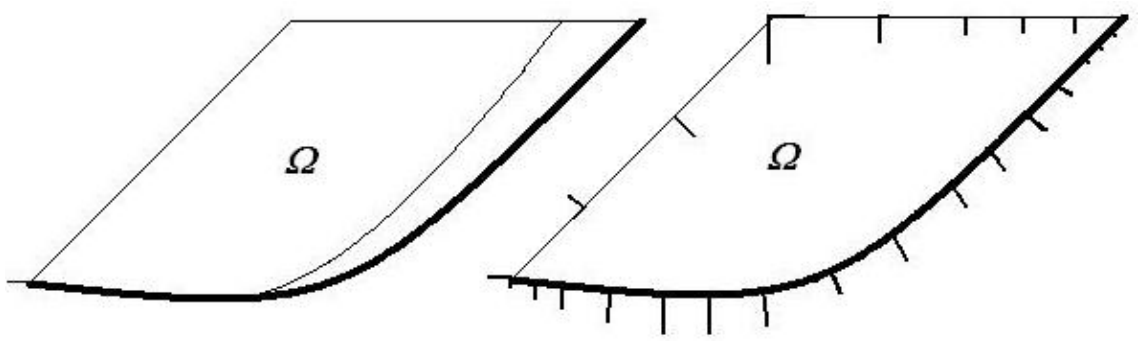

Figure 3: Initial and optimal shape of the slip curve and movements on the optimal slip curve.

\section{Conclusions}

Theoretical background and application of inverse variational principles to the stability of real slopes, which was necessary to assess in a suburb part of urban area of the capital Prague. In this paper it was shown, what is the meaning of optimal slip curve when using the inverse variational principles. It has been proved that the optimal slip curve (i.e. such a slip curve which responds to the minimum stability) is selected according to the condition of uniform distribution 
of contact energy density. This criterion cannot be fulfilled exactly; for example, in the upper part of the slope (close to the ridge) the density of the contact energy must be lower then in the bottom part of the slip curve. This is why the criterion is selected in such a way that the minimum of the energy density is sought along the contact boundary.

The slope of $45^{\circ}$ was concerned in our example. The algorithm allows us to change the slope in such a way that if the current slope is safe enough, steeper slope can be assessed, else the slope has top be decreased. If we use standard PC computer the procedure is very fast. Also the criterion imposed on safety margin can be changed for another one.

\section{Acknowledgment}

Financial support of this research was provided by Grant agency of the Czech Republic, No. 103/06/1124.

\section{References}

[1] Koudelka, P. and Procházka, P. Apriori Integration Method, Analysis, Similarity and Optimization of Slopes. Academia Prague, 2001

[2] Bufler, H. and Horák, V. Die inversen Variationsprinzipien der duennen Platte bei zulassung diskontinuirlicher Dicke, Schnittkraefte und Verschiebungsgroessen. Acta Technikca CSAV, 1970, 271-282

[3] Horák, V. and Procházka, P. Optimal shape design of structures by inverse variational principles. 1st Int. Conf. on Mechanics, CSAV, Prague, Vol. II, 191-194, 1987

[4] Seguchi, Y. and Tada, Y. Shape Determination of Structure Based on the Inverse Variational Principle, the Finite Element Approach, Int. Symp. on Optimal Shape Design, University of Arizona, 1981

[5] Tada, Y., Seguchi, Y. and Soh, T. Shape Determination Problems of Structures by the Inverse Variational Principle, Feasibility Study about Application to Actual Structures. Bulletin of JSME, Vol. 29(253), July 1986

[6] Banerjee, P.K. \& Butterfield, R. Boundary Element Methods in Engineering Science, McGraw-Hill, London, New York 1981

[7] Procházka, P. \& Sejnoha, M. Development of debond region of lag model. Computers \& Structures, Volume 55, Issue 2, 1995, 249-260 